\title{
Application of the COPD Assessment Test (CAT) to Patients with Interstitial Lung Disease
}

\author{
Fujiko Someya*, Takao Nakagawa \\ School of Health Sciences, Kanazawa University, Kanazawa, Japan \\ Email: fujiko@mhs.mp.kanazawa-u.ac.jp
}

Received 2 September 2014; revised 10 October 2014; accepted 31 October 2014

Copyright (C) 2014 by authors and Scientific Research Publishing Inc.

This work is licensed under the Creative Commons Attribution International License (CC BY). http://creativecommons.org/licenses/by/4.0/

(c) (i) Open Access

\section{Abstract}

The COPD Assessment Test was recently developed to assess health status in patients with COPD. However, little was known about its application to patients with interstitial lung disease, so we examined the relationship between the COPD Assessment Test score and respiratory impairment including the clinical picture in subjects with interstitial lung disease. Data were collected retrospectively from 52 consecutive subjects with interstitial lung disease on admission to our facility. All subjects completed the COPD Assessment Test, in which a higher score represented a worse health status. They were also assessed by pulmonary function test, $\mathrm{SpO}_{2}$ during 6-min walk test, and arterial blood gases. The COPD Assessment Test score was negatively correlated with the percentage of predicted forced vital capacity, forced expiratory volume in 1 second, total lung capacity, diffusion capacity of the lung for carbon monoxide, and $\mathrm{PaO}_{2}(p=0.0005,0.005,0.0002$, 0.0005 , and 0.002, respectively). Breathlessness was detected as the high scoring item, while phlegm and sleep were the last affected items. The COPD Assessment Test score was higher in subjects with oxygen desaturation in $\mathrm{SpO}_{2}(\geq 4 \%)$ during 6-min walk test $(p=0.0001)$ and in subjects on home oxygen therapy $(p=0.0007)$. More than $75 \%$ of subjects with oxygen desaturation during walking were classified into a medium or severer impact level of the COPD Assessment Test score, and subjects on home oxygen therapy were classified into a high or severer impact level. $\mathrm{PaCO}_{2}$ in room air and $\mathrm{SpO}_{2}$ at rest did not correlate with the COPD Assessment Test score. The reliability of items in the COPD Assessment Test was 0.87 by Cronbach's $\alpha$ coefficient. Our results suggest that the COPD Assessment Test may be a candidate for evaluating the health status and impact of disease on patients with interstitial lung disease.

\section{Keywords}

Interstitial Lung Disease, Quality of Life, Questionnaire, Pulmonary Function, Oxygen Saturation,

${ }^{*}$ Corresponding author. 


\section{Home Oxygen Therapy}

\section{Introduction}

The health-related quality of life (QOL) in patients with COPD has been assessed using questionnaires such as the Medical Outcomes Study Short-Form 36 [1], St. George’s Respiratory Questionnaire [2]-[4], and the Chronic Respiratory Disease Questionnaire [5] [6]. A correlation was previously reported between QOL and pulmonary function, especially forced expiratory volume in 1 second $\left(\mathrm{FEV}_{1}\right)$ [2] [7]. St. George's Respiratory Questionnaire score was shown to be improved by pulmonary rehabilitation [3] [8] and medication [4]. Moreover, physical impairments including pulmonary function are correlated with preexisting QOL assessment scores in patients with interstitial lung disease using the same questionnaires for COPD [9], and the effectiveness of interventions has been suggested through QOL evaluation [10].

A novel and simple QOL questionnaire was recently developed for use in routine clinical practice to measure the health status of patients with COPD. The results of the COPD Assessment Test (CAT) [11], which only took 107 sec on average to be completed, were correlated with those obtained using St. George's Respiratory Questionnaire, which took an average of 578 sec to be completed [12]. The CAT consists of eight patient-completed items, with higher scores indicating a worse health status, and the total score ranges from 0 to 40 . An increase of approximately 2 points in the score on St. George's Respiratory Questionnaire was shown to correspond to a 1-point increase in the CAT score [13].

The CAT scores were categorized into low, medium, high, and very high impact, and users of the CAT proposed descriptive scenarios, as the clinical picture of COPD and possible management considerations, according to the impact of COPD [14]. A statistically significant relationship between the CAT score and pulmonary function, that is, forced vital capacity (FVC), vital capacity (VC), $\mathrm{FEV}_{1}$, and $\mathrm{PaO}_{2}$, is also shown in patients with interstitial lung disease [15]. However, the clinical picture of the impact of interstitial lung disease by the CAT score has not been elucidated.

Considering the differences in clinical symptoms between COPD and interstitial lung disease, we retrospectively investigated symptoms affecting the CAT score in subjects with interstitial lung disease. Then, we aimed to propose some potential management considerations according to the grading impact by CAT scores like COPD.

\section{Methods}

\subsection{Subjects and Methods}

When examined retrospectively, subject record was anonymized and de-identified prior to analysis. The protocol of the present study was approved by the Human Ethics Committee of the University. Fifty-two consecutive subjects with interstitial lung disease were referred for a closer physical examination at the time of diagnosis or during follow-up when they were admitted to our facility in January 2013 to July 2014 (Table 1). Ten subjects were diagnosed with idiopathic pulmonary fibrosis, 25 with systemic sclerosis, 13 with polymyositis/dermatomyositis, and 4 with rheumatoid arthritis. Five subjects were excluded prior to the selection of subjects because they exhibited severe leg pain or muscle weakness when walking. Interstitial lung disease was confirmed by radiographic and/or histopathological examinations. At the time of inclusion in the study, 12 subjects received home oxygen therapy with a flow rate of up to 3 liters per minute.

Subjects completed the Japanese version of the CAT [13] on the first day of their visit. The eight items in the CAT covered cough, phlegm, chest tightness, breathlessness, activity limitations at home, confidence in leaving the home, sleep, and energy. All subjects could complete a 5-point rating scale of each item by themselves. When the 6-min walk test was performed, $\mathrm{SpO}_{2}$ was recorded at rest and at the end of the test using pulse oximetry. Subjects on home oxygen therapy completed the 6-min walk test with oxygen supplementation at the same oxygen flow rate at home. If subjects could not complete the 6-min walk test due to dyspnea, the $\mathrm{SpO}_{2}$ value was recorded when subjects quit the test. Exercise-induced oxygen desaturation was defined as a decrease of $\geq 4 \%$ from the resting $\mathrm{SpO}_{2}$ value at the end of the walking test [16]-[18]. For this reason, it was reported that a decrease of $\geq 4 \%$ from the resting $\mathrm{SpO}_{2}$ value was significantly associated with the dyspnea index and FVC < 
Table 1. Characteristics of the patients $(n=52)$.

\begin{tabular}{|c|c|}
\hline Variables & Values \\
\hline Age, years & $61.5 \pm 13.7$ \\
\hline Gender f/m & $30 / 22$ \\
\hline Height, cm & $159 \pm 10$ \\
\hline Weight, kg & $57 \pm 12$ \\
\hline Time since diagnosis, years & $8 \pm 9$ \\
\hline CAT total score & $15.5(7.25-22.75)$ \\
\hline Cough & $2(0.25-3)$ \\
\hline Phlegm & $1(0-2)$ \\
\hline Chest tightness & $1.5(0-3)$ \\
\hline Breathlessness & $3(2-5)$ \\
\hline Activity limitation at home & $1(0-3)$ \\
\hline Confidence in leaving the home & $1.5(0-4)$ \\
\hline Sleep & $0(0-2)$ \\
\hline Energy & $2(0.25-3)$ \\
\hline \multicolumn{2}{|l|}{ Pulmonary function } \\
\hline FVC, \% predicted & $84.1 \pm 26.4$ \\
\hline FEV1, \% predicted & $91.2 \pm 22.9$ \\
\hline TLC, \% predicted & $76.6 \pm 21.0$ \\
\hline DLCO, \% predicted & $51.4 \pm 23.9$ \\
\hline $\mathrm{SpO}_{2}$ at rest, $\%$ & $97 \pm 2$ \\
\hline Oxygen desaturation after walking, $\mathrm{n}$ & 29 \\
\hline $\mathrm{PaO}_{2}$ in room air, mmHg & $80.1 \pm 17.2$ \\
\hline $\mathrm{PaCO}_{2}$ in room air, mmHg & $39.4 \pm 5.5$ \\
\hline Home oxygen therapy, n & 12 \\
\hline
\end{tabular}

80\% predicted [18], and implied a risk of mortality [16] in patients with interstitial lung disease.

Clinical information during a closer examination was retrospectively collected from all subjects by reviewing their medical records, in terms of gender, age, morphological measurements, duration of disease at their admission to our facility after diagnosis with interstitial lung disease, percentage of predicted FVC, FEV 1 , total lung capacity (TLC), single-breath diffusing capacity of the lung for carbon monoxide $\left(\mathrm{D}_{\mathrm{LCO}}\right)$ (Chestac-9800, Chest Medical Instruments, Tokyo, Japan), and $\mathrm{PaO}_{2}$ and $\mathrm{PaCO}_{2}$ in room air. $\mathrm{D}_{\mathrm{LCO}}$ was adjusted by the measured hemoglobin concentration [19]; however, $\mathrm{D}_{\mathrm{LCO}}$ could not be measured for eight subjects due to severely impaired pulmonary function.

\subsection{Statistics}

JMP 8 software (SAS Institute Inc., Cary, NC) was used for statistical analysis. The reliability of the eight items in the CAT was examined using Cronbach's $\alpha$ coefficient. Spearman's correlation coefficient was used to examine the correlations between the CAT score and FVC, FEV 1, TLC, DLCO, $\mathrm{SpO}_{2}$ at rest, $\mathrm{PaO}_{2}, \mathrm{and}_{\mathrm{PaCO}}$. Using the Mann-Whitney test, we examined the effects of exercise-induced oxygen desaturation ( $\geq 4 \%$ vs. $<4 \%$ ) and home oxygen therapy (on vs. off) on the CAT scores. Kruskal-Wallis test was used to assess the differences of symptoms in the CAT among the four impact levels of the CAT score. $p<0.05$ was considered significant. 


\section{Results}

The CAT score was negatively correlated with the percentage of predicted FVC, FEV ${ }_{1}$, TLC, $\mathrm{D}_{\mathrm{LCO}}$, and $\mathrm{PaO}_{2}(p$ $=0.0005,0.005,0.0002,0.0005$, and 0.002, respectively) (Table 2). $\mathrm{SpO}_{2}$ at rest was as high as $97 \% \pm 2 \%$ (Table 1) because of oxygen supplementation to subjects on home oxygen therapy; the lowest value of $\mathrm{SpO}_{2}$ was $92 \% . \mathrm{PaCO}_{2}$ was almost normal, and a review of the medical records revealed that no subjects exhibited $\mathrm{CO}_{2}$ narcosis. Consequently, the CAT score did not correlate with $\mathrm{SpO}_{2}$ at rest or $\mathrm{PaCO}_{2}$ (Table 2).

The reliability of the eight items in the CAT was 0.87 by Cronbach's $\alpha$ coefficient, which was markedly higher than 0.70, a value that was considered to be indicative of internal consistency [13]. The scores of all items in the CAT were significantly different among the four impact levels (Table 3). As items were each scored from 0 to 5 , a score of 3 or higher was associated with a likelihood of presenting noticeable symptoms. No median score of items showed a higher score at low impact. A median score of 3 was found for the item of breathlessness, that is, breathlessness when walking up a hill or one flight of stairs, at medium impact. The last items showing a higher median score were phlegm and sleep at very high impact.

The CAT score was significantly higher in subjects with exercise-induced oxygen desaturation than in those without it $(p=0.0001)$ (Figure 1). The 25th percentile score was 13 in subjects with oxygen desaturation. Twelve subjects on home oxygen therapy were included in the group of subjects with exercise-induced oxygen desaturation regardless of oxygen supplementation while walking. These 12 subjects showed significantly higher CAT scores than subjects off home oxygen therapy $(p=0.0007)$, with the 25th percentile score being 21.25 (Figure 2).

Table 2. Spearman's correlation coefficient of the CAT score with parameters of respiratory status $(\mathrm{n}=52)$.

\begin{tabular}{lcc}
\hline Variables & $\mathrm{R}$ & $P$ \\
\hline FVC, \% predicted & -0.47 & 0.0005 \\
FEV1, \% predicted & -0.38 & 0.005 \\
TLC, \% predicted & -0.51 & 0.0002 \\
$\mathrm{D}_{\mathrm{LCO}}$ \% predicted & -0.50 & 0.0005 \\
$\mathrm{SpO}_{2}$ at rest, \% & -0.06 & 0.67 \\
$\mathrm{PaO}_{2}$ in room air, mmHg & -0.48 & 0.002 \\
$\mathrm{PaCO}_{2}$ in room air, mmHg & -0.16 & 0.31 \\
\hline
\end{tabular}

CAT, COPD Assessment Test; FVC, forced vital capacity; $\mathrm{FEV}_{1}$, forced expiratory volume in 1 second; TLC, total lung capacity; $\mathrm{D}_{\mathrm{LCO}}$, diffusion capacity of the lung for carbon monoxide. $\mathrm{D}_{\mathrm{LCO}}$ could not be measured for 8 subjects due to severely impaired pulmonary function.

Table 3. The score of each item in the CAT and impact level.

\begin{tabular}{|c|c|c|c|c|c|}
\hline Impact level & Low & Medium & High & Very High & \multirow{2}{*}{$p$} \\
\hline CAT score & $0-10$ & $11-20$ & $21-30$ & $31-40$ & \\
\hline $\mathrm{N}$ & 19 & 16 & 15 & 2 & \\
\hline Cough & $1(0-2)$ & $2(1-3)$ & $3(0-4)$ & $3.5(3-4)$ & 0.032 \\
\hline Phlegm & $0(0-1)$ & $2(1-2.75)$ & $2(1-4)$ & $4(3-5)$ & 0.003 \\
\hline Chest tightness & $0(0-1)$ & $1.5(0.25-3)$ & $3(2-4)$ & $4(4-4)$ & $<0.0001$ \\
\hline Breathlessness & $1(0-3)$ & $3(2-4)$ & $5(4-5)$ & $5(5-5)$ & $<0.0001$ \\
\hline Activity limitations at home & $0(0-0)$ & $1.5(0.25-2.75)$ & $4(3-5)$ & $3.5(3-4)$ & $<0.0001$ \\
\hline Confidence in leaving the home & $0(0-0)$ & $2(0-3)$ & $4(3-5)$ & $4(4-4)$ & $<0.0001$ \\
\hline Sleep & $0(0-0)$ & $1.5(0-2)$ & $2(0-4)$ & $3(3-3)$ & 0.0002 \\
\hline Energy & $0(0-2)$ & $2.5(2-3)$ & $3(3-4)$ & $4.5(4-5)$ & $<0.0001$ \\
\hline
\end{tabular}

Values are expressed as median (interquartile range). CAT, COPD Assessment Test. Kruskal-Wallis test was used to assess the differences among the four impact levels. 


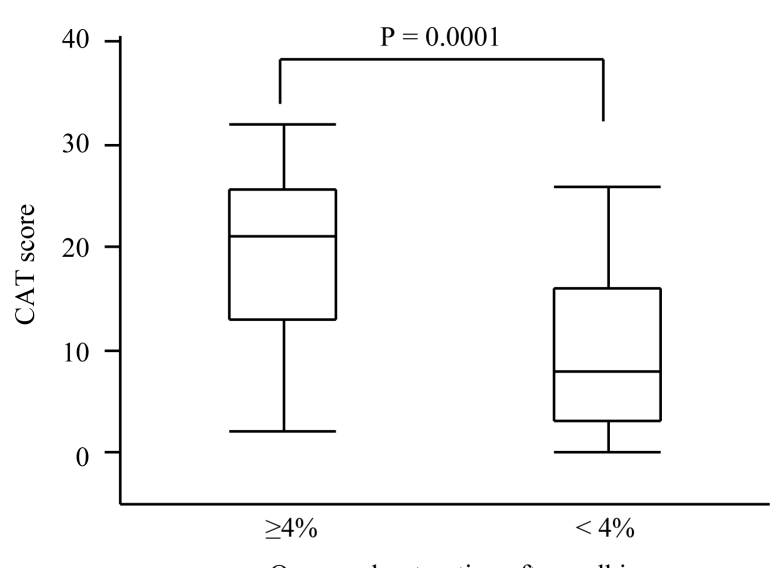

Oxygen desaturation after walking

Figure 1. The COPD Assessment Test (CAT) scores classified according to oxygen desaturation. Boxes represent medians and interquartile ranges, whiskers represent the Tukey boxplots (the lowest datum still within the 1.5 interquartile range of the lower quartile, and the highest datum still within the 1.5 interquartile range of the upper quartile). The 25th percentile score was 13 in subjects with oxygen desaturation.

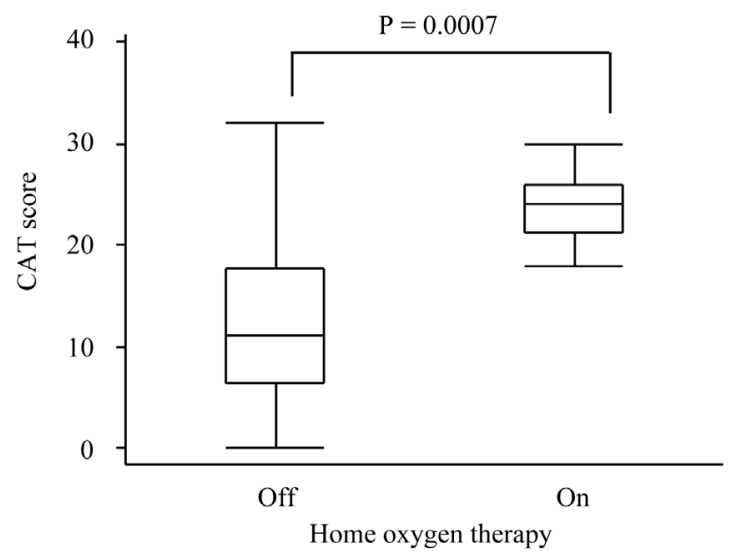

Figure 2. The COPD Assessment Test (CAT) scores classified according to home oxygen therapy. Boxes represent medians and interquartile ranges, whiskers represent the Tukey boxplots (the lowest datum still within the 1.5 interquartile range of the lower quartile, and the highest datum still within the 1.5 interquartile range of the upper quartile). The 25th percentile score was 21.25 in subjects on home oxygen therapy.

\section{Discussion}

In the present study, the CAT score was correlated with percentage of predicted FVC, FEV 1 , TLC, $\mathrm{D}_{\mathrm{LCO}}$, and $\mathrm{PaO}_{2}$. Previously, in patients with interstitial lung disease, components in the Short-Form 36 were correlated with FVC, FEV 1 , and $\mathrm{D}_{\mathrm{LCO}}$, but not with TLC [9] [20]. The Chronic Respiratory Questionnaire score correlated with $\mathrm{D}_{\mathrm{LCO}}$, but not with FVC, $\mathrm{FEV}_{1}$, or TLC [9]]. St. George's Respiratory Questionnaire score correlated with FVC, $\mathrm{FEV}_{1}, \mathrm{D}_{\mathrm{LCO}}$, and TLC [9]. It was also shown that the CAT score correlated with FVC, VC, FEV $\mathrm{F}_{1}$, and $\mathrm{PaO}_{2}$, as mentioned in the introduction [15]. These findings clearly support a significant relationship between QOL evaluation including the CAT score and pulmonary function in patients with interstitial lung disease. In patients with COPD, percentage of predicted $\mathrm{FEV}_{1}$ is an important correlate of QOL among the representatives of pulmonary function [2] [7], and the parameter in patients with interstitial lung disease might also be a good candidate for a primary correlate of QOL. It has been reported that St. George's Respiratory Questionnaire scores were worse for patients with interstitial lung disease than for patients with COPD with similar degrees of $\mathrm{FEV}_{1}$ impairment [21]. The same kind of study would be required for the CAT considering the clinical management of interstitial lung disease. 
As $\mathrm{SpO}_{2}$ at rest was elevated up to $97 \%$ on average, no correlation was observed with the CAT score. However, exercise-induced oxygen desaturation significantly affected the CAT score even in subjects with supplemental oxygen. The 25th percentile score were 13 in patients with exercise-induced oxygen desaturation in the present study, which suggested impairment of oxygen saturation might be of concern at medium and severer impact in interstitial lung disease. Additionally, patients on home oxygen therapy might be at the high and severer impact levels. Previously, prescription of long-term oxygen therapy by an oxygen concentrator was shown not to affect the QOL of patients with severe COPD, although they showed lower QOL than patients with less severe COPD [22]. For this reason, it was explained that COPD patients used oxygen as they expected some benefit in terms of survival. In the present study, the high CAT score in subjects on home oxygen therapy might have been due to the severity of disease and exercise-induced oxygen desaturation.

In the present study, the reliability of items in the CAT as shown by Cronbach's $\alpha$ coefficient was 0.87 . This value was reported as 0.88 in the original CAT [11] and as 0.89 in the Japanese version of the CAT [13], which is the approved translation of the CAT questionnaire for COPD. These results suggest that all items were consistent and measured almost the same underlying construct statistically, even in patients with interstitial lung disease.

Breathlessness was the main symptom for subjects at the medium impact level, scored as 3 . In patients with COPD, breathlessness was part of the clinical picture as well as cough and activity limitations at home at the medium impact level, and these subjects were known to suffer from cough and phlegm [14]. However, cough and activity limitations at home were not noticeable symptoms at the medium impact level (scored as 2 and 1.5, respectively), and phlegm was the last item with a higher score $(\geq 3)$ in subjects in this study. The difference in the clinical picture between interstitial lung disease and COPD was suggested to be shown even by the CAT.

Optimized management should be proposed for patients with a higher CAT score in the future. Pulmonary rehabilitation was suggested to improve QOL in interstitial lung disease [10], so possible management should be considered according to the impact level. For example, pulmonary rehabilitation seemed to improve 6-min walking distance effectively in patients with poor functional status with interstitial lung disease [23]. Therefore, the impact level of the CAT score for beneficial management as pulmonary rehabilitation should be clarified to propose an intervention for patients with interstitial lung disease.

The Japanese version of the CAT was developed in 2012 [13]; therefore, the number of subjects who have taken this test is limited to those who have visited our facility. Even though the number of subjects was small in this study, a significant relationship between the CAT score and pulmonary function was shown. A prospective study is needed to demonstrate the more precise impact of interstitial lung disease, accumulating a broader range of information on the clinical picture and possible management considerations.

\section{Conclusion}

The CAT score in subjects with interstitial lung disease was correlated with pulmonary function and was higher in subjects with exercise-induced oxygen desaturation and on home oxygen therapy than that in those without these factors. The reliability of the eight items in the CAT was as high as that in patients with COPD. Moreover, the clinical symptom found to be the high scoring item was breathlessness. Cough and phlegm, typical symptoms in COPD, were not noticeable at the lower impact level in this study. The CAT might be available to evaluate health-related QOL in patients with interstitial lung disease.

\section{Disclosures}

None.

\section{References}

[1] Mineo, T.C., Ambrogi, V., Pompeo, E., Elia, S., Mineo, D., et al. (2004) Impact of Lung Volume Reduction Surgery versus Rehabilitation on Quality of Life. European Respiratory Journal, 23, 275-280. http://dx.doi.org/10.1183/09031936.03.00025203

[2] Ketelaars, C.A., Schlosser, M.A.G., Mostert, R., Abu-Saad, H.H., Halfens, R.J.G., et al. (1996) Determinants of Health-Related Quality of Life in Patients with Chronic Obstructive Pulmonary Disease. Thorax, 51, 39-43. http://dx.doi.org/10.1136/thx.51.1.39

[3] Foglio, K., Bianchi, L., Bruletti, G., Porta, R., Vitacca, M., et al. (2007) Seven-Year Time Course of Lung Function, 
Symptoms, Health-Related Quality of Life, and Exercise Tolerance in COPD Patients Undergoing Pulmonary Rehabilitation Programs. Respiratory Medicine, 101, 1961-1970. http://dx.doi.org/10.1016/j.rmed.2007.04.007

[4] Spencer, S., Calverley, P.M.A., Burge, P.S. and Jones, P.W. (2001) Health Status Deterioration in Patients with Chronic Obstructive Pulmonary Disease. American Journal Respiratory and Critical Care Medicine, 163, 122-128. http://dx.doi.org/10.1164/ajrccm.163.1.2005009

[5] Wijkstra, P.J., TenVergert, E.M., van der Mark, T.W., Postma, D.S., Altena, R.V., et al. (1994) Relation of Lung Function, Maximal Inspiratory Pressure, Dyspnea, and Quality of Life with Exercise Capacity in Patients with Chronic Obstructive Pulmonary Disease. Thorax, 49, 468-472. http://dx.doi.org/10.1136/thx.49.5.468

[6] Tsai, C.-L., Hodder, R.V., Page, J.H., Cydulka, R.K., Rowe, B.H., et al. (2008) The Short-Form Chronic Respiratory Disease Questionnaire Was Valid, Reliable, and Responsive Quality-of-Life Instrument in Acute Exacerbations of Chronic Obstructive Pulmonary Disease. Journal of Clinical Epidemiology, 61, 489-497. http://dx.doi.org/10.1016/j.jclinepi.2007.07.003

[7] Mahler, D.A., Faryniarz, K., Tomlinson, D., Colice, G.L., Robins, A.G., et al. (1992) Impact of Dyspnea and Physiologic Function on General Health Status in Patients with Chronic Obstructive Pulmonary Disease. Chest, 102, 395401. http://dx.doi.org/10.1378/chest.102.2.395

[8] Laviolette, L., Bourbeau, J., Bernard, S., Lacasse, Y., Pepin, V., et al. (2008) Assessing the Impact of Pulmonary Rehabilitation of Functional Status in COPD. Thorax, 63, 115-121. http://dx.doi.org/10.1136/thx.2006.076844

[9] Chang, J.A., Curtis, J.R., Patrick, D.L. and Raghu, G. (1999) Assessment of Health-Related Quality of Life in Patients with Interstitial Lung Disease. Chest, 116, 1175-1182. http://dx.doi.org/10.1378/chest.116.5.1175

[10] Bajwah, S., Ross, J.R., Peacock, J.L., Higginson, I.J., Wells, A.U., et al. (2013) Interventions to Improve Symptoms and Quality of Life of Patients with Fibrotic Interstitial Lung Disease: A Systematic Review of the Literature. Thorax, 68, 867-879. http://dx.doi.org/10.1136/thoraxjnl-2012-202040

[11] Jones, P.W., Harding, G., Berry, P., Wiklund, I., Chen, W.H. and Kline Leidy, N. (2009) Development and First Validation of the COPD Assessment Test. European Respiratory Journal, 34, 648-654. http://dx.doi.org/10.1183/09031936.00102509

[12] Ringbaek, T., Martinez, G. and Lange, P. (2012) A Comparison of the Assessment of Quality of Life with CAT, CCQ, and SGRQ in COPD Patients Participating in Pulmonary Rehabilitation. COPD: Journal of Chronic Obstructive Pulmonary Disease, 9, 12-15. http://dx.doi.org/10.3109/15412555.2011.630248

[13] Tsuda, T., Suematsu, R., Kamohara, K., Kurobe, M., Arakawa, I., Tomioka, R., et al. (2012) Development of the Japanese Version of the COPD Assessment Test. Respiratory Investigation, 50, 34-39. http://dx.doi.org/10.1016/j.resinv.2012.05.003

[14] Jones, P.W., Tabberer, M. and Chen, W.H. (2011) Creating Scenarios of the Impact of COPD and Their Relationship to COPD Assessment Test (CAT) Scores. BMC Pulmonary Medicine, 11, 42. http://www.biomedcentral.com/1471-2466/11/42 http://dx.doi.org/10.1186/1471-2466-11-42

[15] Nagata, K., Tomii, K., Otsuka, K., Tachikawa, R., Otsuka, K., Takeshita, J., et al. (2012) Evaluation of the Chronic Obstructive Pulmonary Disease Assessment Test for Measurement of Health-Related Quality of Life in Patients with Interstitial Lung Disease. Respirology, 17, 506-512. http://dx.doi.org/10.1111/j.1440-1843.2012.02131.X

[16] Lama, V.N., Flaherty, K.R., Toews, G.B., Colby, T.V., Travis, W.D., Long, Q., et al. (2003) Prognostic Value of Desaturation during a 6-Minute Walk Test in Idiopathic Interstitial Pneumonia. American Journal of Respiratory and Critical Care Medicine, 168, 1084-1090. http://dx.doi.org/10.1164/rccm.200302-219OC

[17] Poulain, M., Durand, F., Palomba, B., Ceugniet, F., Desplan, J., Varray, A. and Préfaut, C. (2003) 6-Minute Walk Testing in More Sensitive than Maximal Incremental Cycle Testing for Detecting Oxygen Desaturation in Patients with COPD. Chest, 123, 1401-1407. http://dx.doi.org/10.1378/chest.123.5.1401

[18] Villalba, W.O., Sampaio-Barros, P.D., Pereira, M.C., Cerqueira, E.M.F.P., Leme Jr., C.A., Marques-Neto, J.F. and Paschoal, I.A. (2007) Six-Minute Walk Test for the Evaluation of Pulmonary Disease Severity in Scleroderma Patients. Chest, 131, 217-222. http://dx.doi.org/10.1378/chest.06-0630

[19] Wallaert, B., Wemeau-Stervinou, L., Salleron, J., Tillie-Leblond, I. and Perez, T. (2012) Do We Need Exercise Tests to Detect Gas Exchange Impairment in Fibrotic Idiopathic Interstitial Pneumonias? Pulmonary Medicine, 2012, Article ID: 657180. http://dx.doi.org/10.1155/2012/657180

[20] Martinez, T.Y., Pereira, C.A., dos Santos, M.L., Ciconelli, R.M., Guimaraes, S.M. and Martinez, J.A.B. (2000) Evaluation of the Short-Form 36-Item Questionnaire to Measure Health-Related Quality of Life in Patients with Idiopathic Pulmonary Fibrosis. Chest, 117, 1627-1632. http://dx.doi.org/10.1378/chest.117.6.1627

[21] Berry, C.E., Drummond, M.B., Han, M.K., Li, D., Fuller, C., Limper, A.H., et al. (2012) Relationship between Lung Function Impairment and Health-Related Quality of Life in COPD and Interstitial Lung Disease. Chest, 142, 704-711. 
http://dx.doi.org/10.1378/chest.11-1332

[22] Okubadejo, A.A., Paul, E.A., Jones, P.W. and Wedzicha, J.A. (1996) Does Long-Term Oxygen Therapy Affect Quality of Life in Patients with Chronic Obstructive Pulmonary Disease and Severe Hypoxaemia? European Respiratory Journal, 9, 2335-2339. http://dx.doi.org/10.1183/09031936.96.09112335

[23] Ferreira, A., Barvey, C., Connors, G.L., Hilling, L., Rigler, J., Farrell, S., et al. (2009) Pulmonary Rehabilitation in Interstitial Lung Disease: Benefits and Predictors of Response. Chest, 135, 442-447.

http://dx.doi.org/10.1378/chest.08-1458 
Scientific Research Publishing (SCIRP) is one of the largest Open Access journal publishers. It is currently publishing more than 200 open access, online, peer-reviewed journals covering a wide range of academic disciplines. SCIRP serves the worldwide academic communities and contributes to the progress and application of science with its publication.

Other selected journals from SCIRP are listed as below. Submit your manuscript to us via either submit@scirp.org or Online Submission Portal.
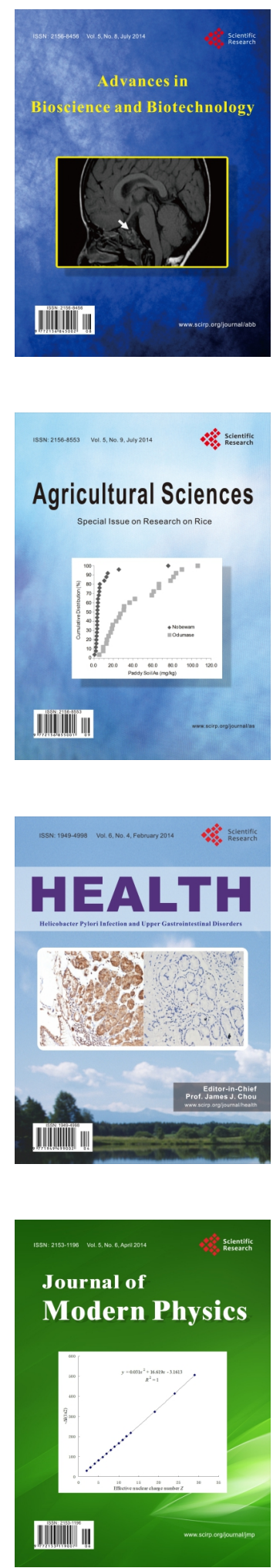
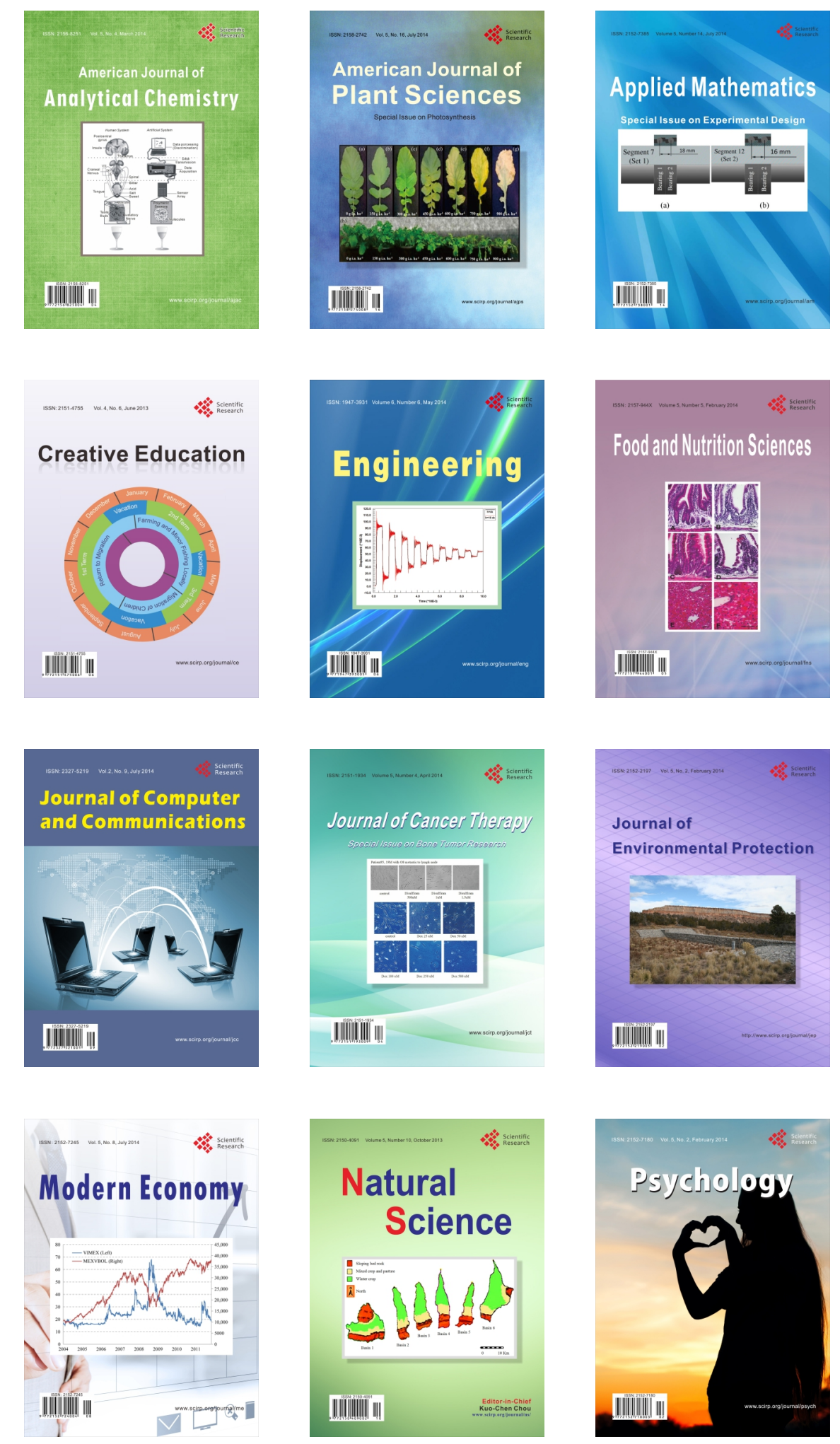